\title{
HITTING TIMES, OCCUPATION TIMES, TRIVARIATE LAWS AND THE FORWARD KOLMOGOROV EQUATION FOR A ONE-DIMENSIONAL DIFFUSION WITH MEMORY
}

\author{
MARTIN FORDE ${ }^{* * *}$ AND \\ ANDREY POGUDIN, ${ }^{* * * *}$ King's College London \\ HONGZHONG ZHANG, ${ }^{* * * *}$ Columbia University
}

\begin{abstract}
We extend many of the classical results for standard one-dimensional diffusions to a diffusion process with memory of the form $\mathrm{d} X_{t}=\sigma\left(X_{t}, \underline{X}_{t}\right) \mathrm{d} W_{t}$, where $\underline{X}_{t}=$ $m \wedge \inf _{0 \leq s \leq t} X_{s}$. In particular, we compute the expected time for $X$ to leave an interval, classify the boundary behavior at 0 , and derive a new occupation time formula for $X$. We also show that $\left(X_{t}, \underline{X}_{t}\right)$ admits a joint density, which can be characterized in terms of two independent tied-down Brownian meanders (or, equivalently, two independent Bessel-3 bridges). Finally, we show that the joint density satisfies a generalized forward Kolmogorov equation in a weak sense, and we derive a new forward equation for downand-out call options.
\end{abstract}

Keywords: One-dimensional diffusion; occupation time formula; stochastic functional differential equation; diffusion with memory

2010 Mathematics Subject Classification: Primary 60J60; 47D07

\section{Introduction}

In [10] Forde constructed a weak solution to the stochastic functional differential equation $X_{t}=x+\int_{0}^{t} \sigma\left(X_{s}, M_{s}\right) \mathrm{d} W_{s}$, where $M_{t}=\sup _{0 \leq s \leq t} X_{s}$. Using excursion theory, Forde then solved the following problem: for a natural class of joint density functions $\mu(y, b)$, specify $\sigma(\cdot, \cdot)$ so that $X$ is a martingale, and the terminal level and supremum of $X$, when stopped at an independent exponential time $\xi_{\lambda}$, is distributed according to $\mu$. The proof uses excursion theory for regular diffusions to compute an explicit expression for the Laplace transform of the joint density of the terminal level and the supremum of $X$ at an independent exponential time, and the joint density satisfies a forward Kolmogorov equation. Integrating twice, Forde obtained a forward partial differential equation for the up-and-out put option payoff which was then used to back out $\sigma$ from the pre-specified joint density. This was inspired by the earlier work of Carr [5] and Cox et al. [6], who showed how to construct a one-dimensional diffusion with a given marginal at an independent exponential time.

The main result, Theorem 3.6 of [4], shows that we can match the joint distribution at each fixed time of various functionals of an Itô process, including the maximum-to-date or the running average of one component of the Itô process. The mimicking process is also a weak solution to the stochastic functional differential equation (SFDE) and in the special case when

Received 5 July 2012; revision received 21 October 2012.

* Postal address: Department of Mathematics, King's College London, Strand, London WC2R 2LS, UK.

** Email address: martin.forde@kcl.ac.uk

*** Email address: andrey.pogudin@kcl.ac.uk

**** Postal address: Department of Statistics, Columbia University, New York, NY 10027, USA.

Email address: hzhang@stat.columbia.edu 
we are mimicking the terminal level and the maximum, the mimicking process is of the form $X_{t}=x+\int_{0}^{t} \sigma\left(X_{s}, M_{s}, s\right) \mathrm{d} W_{s}$.

In this article we consider the case when the diffusion coefficient $\sigma(\cdot)$ depends only on $X$ and its running minimum, and we assume that $X$ is strictly positive, and $\sigma(x, m)$ is continuous with $0<\sigma(x, m)<\infty$ for $x>0$ and $m \geq 0, m \leq x$, and that $\sigma(0,0)=0$. The purpose of the article is to extend many of the standard well-known results for one-dimensional diffusions to the case when $\sigma$ also depends on the running minimum (as opposed to solving one problem in particular), and we give financial motivation/applications where appropriate.

In Theorem 2.1 we prove weak existence and uniqueness in law for $\mathrm{d} X_{t}=\sigma\left(X_{t}, \underline{X}_{t}\right) \mathrm{d} W_{t}$ by extending the usual time-change argument for one-dimensional diffusions. In Proposition 3.1, we compute the expected length of time to hit either of two barriers for $X$, as a simple application of Itô's lemma and the optional sampling theorem. We then examine the nontrivial question of when the hitting time $H_{0}$ to 0 is finite or not (almost surely); specifically, in Theorem 4.1 we show that, for $\varepsilon \in(0, m)$,

$$
\mathbb{P}\left(H_{0}<\infty\right)=0 \text { if and only if } \int_{0}^{\varepsilon} \int_{0}^{u} \tilde{m}(u, v) \mathrm{d} v \mathrm{~d} u=\infty,
$$

where $\tilde{m}(x, m)=1 / \sigma(x, m)^{2}$. For the case when $\tilde{m}$ is independent of $m$, this reduces to the well-known condition that $\mathbb{P}\left(H_{0}<\infty\right)=0$ if and only if $\int_{0}^{\varepsilon} v \tilde{m}(v) \mathrm{d} v=\infty$ (see, e.g. Theorem 51.2(i) of [21]). We then formulate an extension of the classical occupation time formula for the new $X$ process (Theorem 5.1).

In Theorem 6.1, by adapting the argument in [18], using Girsanov's theorem, and conditioning on the terminal value and the minimum of $X$, we prove the existence of the joint density $p_{t}(x, m)$ for $X$ and its minimum. We then further characterize this joint density in terms of two independent back-to-back Brownian meander bridges, which we can further represent in terms of two independent Bessel-3 bridges using standard results in, e.g. [1], [2], and [11]. Finally, in Section 8 we show that $X$ is a weak solution to a forward Kolmogorov equation, and we also derive a new forward equation for down-and-out call options.

\section{A one-dimensional diffusion with memory}

In this section we construct a weak solution to the SFDE

$$
X_{t}=x+\int_{0}^{t} \sigma\left(X_{s}, \underline{X}_{s}\right) \mathrm{d} W_{s},
$$

where $\underline{X}_{t}=m \wedge \inf _{0 \leq s \leq t} X_{s}$ and $W$ is a standard Brownian motion, and we show that the solution $X$ is unique in law. The $m$ parameter allows us to include the possibility that $X$ has accrued a previous historical minimum $m$ which may be less than $X_{0}=x$.

We make the following assumptions on $\sigma$ throughout.

Assumption 2.1. (i) $\sigma$ is continuous, and strictly positive away from $(0,0)$.

(ii) $\sigma(0,0)=0$.

(iii) $\lim _{x \searrow 0} x / \sigma(x, x)^{2}=0$.

We let $H_{b}$ denote the first hitting time to $b$, i.e.

$$
H_{b}=\inf \left\{s: X_{s}=b\right\},
$$

and define $\tilde{m}(u, v)=1 / \sigma(u, v)^{2}$. 


\subsection{Weak existence and uniqueness in law}

Theorem 2.1. Equation (2.1) has a nonexploding weak solution for $t<H_{\delta}$ which is unique in law, where $0<\delta \leq m \leq x$.

Proof. Existence. Let $\left(B_{t}, P_{x}\right)$ denote a standard Brownian motion defined on some $(\Omega, \mathcal{F}$, $\left(\mathcal{F}_{t}\right)$ ) with $B_{0}=x>0$ and $\underline{B}_{t}=\inf _{0 \leq s \leq t} B_{s}$, and assume that $\mathcal{F}_{t}$ satisfies the usual conditions (i.e. $\mathcal{F}_{t}$ is right continuous and $\mathcal{F}_{0}$ contains all $\mathcal{F}$ sets of measure 0 ). Let $T_{t}$ denote the almost surely (a.s.), strictly increasing process

$$
T_{t}=\int_{0}^{t} \tilde{m}\left(B_{s}, m \wedge \underline{B}_{s}\right) \mathrm{d} s
$$

for $t<\tau_{\delta}$ and some $\delta>0$, where

$$
\tau_{a}=\inf \left\{s: B_{s}=a\right\} .
$$

Let $A_{t}=\inf \left\{s: T_{s}=t\right\}$ denote the inverse of $T_{t}$, and set

$$
X_{t}=B_{A_{t}} \text {. }
$$

Then we have

$$
\int_{0}^{A_{t}} \sigma^{2}\left(B_{s}, m \wedge \underline{B}_{s}\right) \mathrm{d} T_{s}=\int_{0}^{A_{t}} \mathrm{~d} s=A_{t} .
$$

If we make the change of variables $u=T_{s}$ so $\mathrm{d} u=\mathrm{d} T_{s}=\tilde{m}\left(B_{s}, m \wedge \underline{B}_{s}\right) \mathrm{d} s$ then we can rewrite the integral on the left-hand side as

$$
A_{t}=\int_{0}^{t} \sigma^{2}\left(X_{u}, \underline{X}_{u}\right) \mathrm{d} u \quad \text { a.s. }
$$

where we have used a pathwise application of the Lebesgue-Stieltjes change-of-variable formula. Thus, $\langle X\rangle_{t}=A_{t}$ a.s. Then, by Theorem 3.4.2 of [12], there exists a Brownian motion $W$ on some extended probability space such that (2.1) is satisfied.

Uniqueness in law. We proceed along similar lines to Lemma V.28.7 of [21]. By Theorem IV.34.11 of [21], if $X$ satisfies (2.1) then

$$
B_{t}=X_{T_{t}}
$$

is standard Brownian motion, where $T_{t}=\inf \left\{s:\langle X\rangle_{s}=t\right\}$, so

$$
\int_{0}^{T_{t}} \sigma\left(X_{s}, \underline{X}_{s}\right)^{2} \mathrm{~d} s=t .
$$

Differentiating with respect to $t$ we obtain

$$
\sigma\left(X_{T_{t}}, \underline{X}_{T_{t}}\right)^{2} T_{t}^{\prime}=1=\sigma\left(B_{t}, m \wedge \underline{B}_{t}\right)^{2} T_{t}^{\prime},
$$

where $T_{t}^{\prime}=\tilde{m}\left(B_{t}, m \wedge \underline{B}_{t}\right)$. Hence,

$$
\langle X\rangle_{t}=\inf \left\{u: \int_{0}^{u} \tilde{m}\left(B_{s}, m \wedge \underline{B}_{s}\right) \mathrm{d} s=t\right\} .
$$

Thus, $X$ may be described explicitly in terms of the Brownian motion $B$, so the law of $X$ is uniquely determined.

Finally, stopping $X$ at $H_{\delta}$ means we are only running $B$ until time $\tau_{\delta}$, and $\tau_{\delta}<\infty$ a.s., so $\left(X_{t \wedge H_{\delta}}\right)$ cannot explode to $\infty$ a.s.

From here on we work on the canonical sample space $\Omega=C\left([0, \infty), \mathbb{R}^{+}\right)$with the canonical process $X_{t}(\omega)=\omega(t)(\omega \in \Omega, t \in[0, \infty))$ and its canonical filtration $\mathcal{F}_{t}=\sigma\left(X_{s} ; s \leq t\right)$. 
Let $\mathbb{P}_{x, m}$ denote the law on $(\Omega, \mathcal{B}(\Omega))$ induced by a weak solution to (2.1) (which is unique by Theorem 2.1).

Remark 2.1. If $\sigma \equiv \sigma(x, m, t)$ is time dependent, we can still obtain weak existence and uniqueness if the solution to the ordinary differential equation $\mathrm{d} T_{t}=\tilde{m}\left(B_{t}, m \wedge \underline{B}_{t}, T_{t}\right) \mathrm{d} t$ is uniquely determined a.s. This will be the case if $\tilde{m}$ is Lipschitz in the third argument (we thank Gerard Brunick for pointing this out).

We refer the reader to [14] and [15] for existence and uniqueness results for general SFDEs.

\subsection{Application in financial modelling}

We consider a time-homogeneous local volatility model with memory for a forward price process $\left(F_{t}\right)_{t \geq 0}$ which satisfies

$$
\mathrm{d} F_{t}=F_{t} \mu \mathrm{d} t+F_{t} \sigma\left(F_{t}, \underline{F}_{t}\right) \mathrm{d} W_{t}
$$

under the physical measure $\mathbb{P}$. This has the desirable feature of being a complete model, so, under the unique risk neutral measure $\mathbb{Q}, F_{t}$ will satisfy $\mathrm{d} F_{t}=F_{t} \sigma\left(F_{t}, \underline{F}_{t}\right) \mathrm{d} W_{t}$, i.e. a diffusion-type process of the form in (2.1).

\section{The expected time to leave an interval}

The following proposition computes a closed-form expression for the expectation of the exit time from an interval, using Itô's lemma and a simple application of the optional sampling theorem. This proposition will be needed in the next section where we classify the boundary behaviour of $X$ at 0 . The proof is similar to that used for a regular diffusion in Section 5.5, part C of [12] and [13, p. 197].

Proposition 3.1. The expected time for $X$ to leave the interval $(a, b)$ is given by

$$
\begin{aligned}
h(x, m) & =\mathbb{E}_{x, m}\left(H_{a} \wedge H_{b}\right) \\
& =2 \int_{m}^{x}(u-x) \tilde{m}(u, m) \mathrm{d} u+\frac{2(x-m)}{b-m} \int_{m}^{b}(b-u) \tilde{m}(u, m) \mathrm{d} u+2(b-x) C(m) \\
& <\infty
\end{aligned}
$$

for $0<a \leq m \leq x \leq b<\infty$, where

$$
C(m)=\int_{a}^{b} \int_{a}^{u \wedge m} \frac{b-u}{(b-v)^{2}} \tilde{m}(u, v) \mathrm{d} v \mathrm{~d} u .
$$

Proof. We can easily verify that $h(x, m)$ satisfies

$$
\tilde{m}(x, m)=-\frac{1}{2} h_{x x}, \quad h_{m}(m, m)=0,
$$

with endpoint condition $h(a, a)=h(b, m)=0$ for all $a \leq m<b$.

Now let $\tau=H_{a} \wedge H_{b}$. Then by Itô's lemma we have

$$
\begin{aligned}
h\left(X_{t \wedge \tau}, \underline{X}_{t \wedge \tau}\right)-h(x, m)= & \int_{0}^{t \wedge \tau} h_{x}\left(X_{s}, \underline{X}_{s}\right) \mathrm{d} X_{s}+\frac{1}{2} \int_{0}^{t \wedge \tau} h_{x x}\left(X_{s}, \underline{X}_{s}\right) \sigma^{2}\left(X_{s}, \underline{X}_{s}\right) \mathrm{d} s \\
& +\int_{0}^{t \wedge \tau} h_{m}\left(X_{s}, \underline{X}_{s}\right) \mathrm{d} \underline{X}_{s} \\
= & \int_{0}^{t \wedge \tau} h_{x}\left(X_{s}, \underline{X}_{s}\right) \mathrm{d} X_{s}+\frac{1}{2} \int_{0}^{t \wedge \tau} h_{x x}\left(X_{s}, \underline{X}_{s}\right) \sigma^{2}\left(X_{s}, \underline{X}_{s}\right) \mathrm{d} s
\end{aligned}
$$


using the second equation in (3.2) and the fact that $\mathrm{d} \underline{X}_{t}=0$ if $X_{t} \neq \underline{X}_{t}$. The functions $h_{x}(u, v)$ and $\sigma(u, v)$ are bounded for $0<a \leq v \leq u \leq b$, so taking expectations and applying the optional sampling theorem, and using the first equation in (3.2) yields

$$
\mathbb{E}_{x, m}\left(h\left(X_{t \wedge \tau}, \underline{X}_{t \wedge \tau}\right)\right)=h(x, m)-\mathbb{E}_{x, m}(t \wedge \tau) .
$$

Let $\tilde{m}(u, v) \leq K$ for $0<a \leq v \leq u \leq b$ and some constant $K>0$, so we have

$$
\begin{aligned}
h(x, m) & =\mathbb{E}_{x, m}\left(H_{a} \wedge H_{b}\right) \\
& =2 \int_{m}^{x}(u-x) \tilde{m}(u, m) \mathrm{d} u+\frac{2(x-m)}{b-m} \int_{m}^{b}(b-u) \tilde{m}(u, m) \mathrm{d} u+2(b-x) C(m) \\
& \leq 2 K\left[\int_{m}^{x}(x-u) \mathrm{d} u+\int_{m}^{b}(b-u) \mathrm{d} u+(b-x) \int_{a}^{b} \int_{a}^{u \wedge m} \frac{b-u}{(b-v)^{2}} \mathrm{~d} v \mathrm{~d} u\right] \\
& <\infty
\end{aligned}
$$

Thus, $h(\cdot, \cdot)$ is continuous and bounded; so letting $t \rightarrow \infty$ in (3.3), applying the dominated convergence theorem to the left-hand side and the monotone convergence theorem to the righthand side, and using the fact that $h(a, a)=h(b, m)=0$, we obtain (3.1).

\section{Absorption at zero}

Theorem 4.1. Let $\varepsilon \in(0, m)$. Then we have the following boundary behaviour for $X$ :

$$
\mathbb{P}_{x, m}\left(H_{0}<\infty\right)=0 \text { if and only if } \int_{0}^{\varepsilon} \int_{0}^{u} \tilde{m}(u, v) \mathrm{d} v \mathrm{~d} u=\infty .
$$

Remark 4.1. For the case when $\tilde{m}$ is independent of $m, X$ is a regular one-dimensional diffusion, and Theorem 4.1 reduces to the well-known condition that

$$
\mathbb{P}_{x}\left(H_{0}<\infty\right)=0 \text { if and only if } \int_{0+} v \tilde{m}(v) \mathrm{d} v=\infty
$$

(see, e.g. Theorem 51.2(i) of [21]).

Proof of Theorem 4.1. Setting $a=0$ in (3.1), we have

$$
C(m)=\int_{0}^{b} \int_{a}^{u \wedge m} \frac{b-u}{(b-v)^{2}} \tilde{m}(u, v) \mathrm{d} v \mathrm{~d} u
$$

and $\mathbb{E}_{x, m}\left(H_{0} \wedge H_{b}\right)<\infty$ if and only if $C(m)<\infty$, because $\tilde{m}(0,0)=\infty$ and $\tilde{m}<\infty$ elsewhere, all the upper limits of integration are finite, and $1 /(b-v)$ will not explode because the upper range of $v$ is $m<b$. Noting that $(b-u) /(b-v)^{2} \rightarrow 1$ as $u, v \searrow 0$ and replacing the upper limits of integration by $\varepsilon \in(0, m)$, we see that

$$
\mathbb{E}_{x, m}\left(H_{0} \wedge H_{b}\right)<\infty \text { if and only if } C^{\varepsilon}(m)=\int_{0}^{\varepsilon} \int_{a}^{u \wedge m} \tilde{m}(u, v) \mathrm{d} v \mathrm{~d} u<\infty .
$$

Thus, we have established that $\mathbb{E}_{x, m}\left(H_{0} \wedge H_{b}\right)<\infty$ if and only if $\int_{0}^{\varepsilon} \int_{0}^{u} \tilde{m}(u, v) \mathrm{d} u \mathrm{~d} v<\infty$. We now need to verify that $\mathbb{P}_{x, m}\left(H_{0}<\infty\right)=0$ if and only if $\int_{0}^{\varepsilon} \int_{0}^{u} \tilde{m}(u, v) \mathrm{d} v \mathrm{~d} u=\infty$.

First assume that $\int_{0}^{\varepsilon} \int_{0}^{u} \tilde{m}(u, v) \mathrm{d} v \mathrm{~d} u<\infty$. Then $\mathbb{E}_{x, m}\left(H_{0} \wedge H_{b}\right)<\infty$, so $H_{0} \wedge H_{b}<\infty$ a.s. and $\mathbb{P}_{x, m}\left(H_{0}=H_{b}=\infty\right)=0$. But, from the construction of $X$ via the time-changed 
Brownian motion $B$ in (2.4), we know that $\mathbb{P}_{x}\left(\tau_{0}<\tau_{b}\right)>0$, where $\tau_{a}$ is the first hitting time of $B$ to $a$ as defined in (2.3); hence, $\mathbb{P}_{x, m}\left(H_{0} \leq H_{b}\right)>0, \mathbb{P}_{x, m}\left(H_{0}<H_{b}\right)>0$, and

$$
\mathbb{P}_{x, m}\left(H_{0}<\infty\right) \geq \mathbb{P}_{x, m}\left(H_{0}<H_{b} \leq \infty\right)>0 .
$$

Conversely, assume that $\mathbb{P}_{x, m}\left(H_{0}<\infty\right)>0$. For this part, we proceed as in the proof of Lemma 6.2 of [13]. Then there exists a $t>0$ for which

$$
\mathbb{P}_{x, m}\left(H_{0}<t\right)=\alpha>0 .
$$

Every path starting at $x$ and reaching 0 prior to time $t$ visits every intervening state $\xi \in(0, x)$. Thus, we have

$$
0 \leq \alpha \leq \mathbb{P}_{x, m}\left(H_{0}-H_{\xi}<t\right)=\mathbb{P}_{\xi, \xi \wedge m}\left(H_{0}<t\right) \leq \mathbb{P}_{\xi, \xi \wedge m}\left(H_{x} \wedge H_{0}<t\right)
$$

for $0<\xi \leq x$. It follows that

$$
\sup _{\xi \in(0, x]} \mathbb{P}_{\xi,, \xi \wedge m}\left(H_{x} \wedge H_{0} \geq t\right) \leq 1-\alpha<1,
$$

and by induction we find that

$$
\sup _{\xi \in(0, x)} \mathbb{P}_{\xi, \xi \wedge m}\left(H_{x} \wedge H_{0} \geq n t\right) \leq(1-\alpha)^{n}<1
$$

We can rewrite this as

$$
\mathbb{P}_{\xi, \xi \wedge m}\left(H_{x} \wedge H_{0} \geq a\right) \leq(1-\alpha)^{[a / t]} \leq(1-\alpha)^{a / t-1} .
$$

We now recall the general result in, e.g. [24, p. 79]. For any nonnegative random variable $Y$, we have

$$
\mathbb{E}(Y)=\int_{[0, \infty)} \mathbb{P}(Y \geq y) \mathrm{d} y .
$$

Thus, $\mathbb{E}(Y)<\infty$ if and only if $\int_{(R, \infty)} \mathbb{P}(Y \geq y) \mathrm{d} y<\infty$ for any $R>0$. Thus, setting $Y=H_{x} \wedge H_{0}$ we have

$$
\mathbb{E}_{\xi, \xi \wedge m}\left(H_{x} \wedge H_{0}\right)<\infty \text { if and only if } \int_{[R, \infty)} \mathbb{P}_{\xi, \xi \wedge m}\left(H_{x} \wedge H_{0} \geq a\right) \mathrm{d} a<\infty
$$

But, from (4.1) we have

$$
\int_{[R, \infty)} \mathbb{P}_{\xi, \xi \wedge m}\left(H_{x} \wedge H_{0} \geq a\right) \mathrm{d} a \leq \int_{R}^{\infty}(1-\alpha)^{a / t-1} \mathrm{~d} a=\frac{t(1-\alpha)^{-1+R / t}}{\log (1-\alpha)}<\infty .
$$

Thus, $\mathbb{E}_{\xi, \xi \wedge m}\left(H_{x} \wedge H_{0}\right)<\infty$, and from the first part of the proof we know that $\mathbb{E}_{\xi, \xi \wedge m}\left(H_{x} \wedge H_{0}\right)$ is finite if and only if $\int_{0}^{\varepsilon} \int_{0}^{u} \tilde{m}(u, v) \mathrm{d} v \mathrm{~d} u<\infty$ for all $\varepsilon \leq m$.

Remark 4.2. For a stock price model of the form in (2.2), Theorem 4.1 allows us to compute whether or not the stock will default by hitting 0 or not in a finite time under the risk neutral measure $\mathbb{Q}$, which is relevant for the pricing of so-called credit default swaps, which pay 1 dollar at maturity $T$ if the stock defaults before $T$. 


\section{The occupation time formula}

From the continuity of $\sigma$, we see that, for any $R \in(1, \infty)$ and $0<1 / R \leq v \leq u<R$, $\tilde{m}(u, v)$ is continuous in $v$, and, thus (by the Heine-Cantor theorem), is uniformly continuous in $v$ on the compact set $0<1 / R \leq v \leq u<R$ with fixed $v$. Using this property, we will construct an approximating sequence of processes $\left(X^{n}\right)$ to the process $X$ in (2.1) by 'freezing' the $m$-dependence on a small interval. We then derive a new occupation time formula for $X$ by applying the standard occupation time formula for regular diffusions to the approximating process on each small interval, and then letting $n \rightarrow \infty$.

\subsection{Almost-sure convergence for an approximating sequence of diffusion processes}

Recall that $\tau_{b}=\inf \left\{s: B_{s}=b\right\}$. Set $0<b \leq m \leq x$ and $\tilde{m}_{n}(u, v)=\tilde{m}(u,[v n] / n)$ for $n \geq 1$, so that $\tilde{m}_{n}(u, v)$ is piecewise constant in $v$, and define the process

$$
X_{t}^{n}=B_{A_{t}^{n}}
$$

where $A_{t}^{n}$ is the strictly increasing continuous inverse of

$$
T_{t}^{n}=\int_{0}^{\tau_{m} \wedge t} \tilde{m}\left(B_{s}, m\right) \mathrm{d} s+\int_{\tau_{m} \wedge t}^{t} \tilde{m}_{n}\left(B_{s}, \underline{B}_{s}\right) \mathrm{d} s
$$

for $0 \leq t<\tau_{0}$. Note that $X_{t}=X_{t}^{n}$ for $0 \leq t \leq H_{m}$, because the $m$ dependence in $\sigma$ is 'frozen' until $X$ sets a new minimum below $m$.

Proposition 5.1. Let $H_{b}^{n}=\inf \left\{s: X_{s}^{n}=b\right\}$ and $H_{b}=\inf \left\{s: X_{s}=b\right\}$ as before for $b \in$ $(0, m)$. Then $H_{b}^{n} \rightarrow H_{b}$ a.s. and $X_{t \wedge H_{b}}-X_{t \wedge H_{b}^{n}}^{n} \rightarrow 0$ a.s.

Proof. Without loss of generality, we assume that $x=m$; otherwise, we just start from time $H_{m}$ instead of time 0 . From the time-change construction in the proof of Theorem 2.1, we know that $B_{t}=X_{T_{t}}$ and $B_{\tau_{b}}=X_{H_{b}}$, so we have

$$
H_{b}=\int_{0}^{\tau_{b}} \tilde{m}\left(B_{s}, \underline{B}_{s}\right) \mathrm{d} s
$$

and, similarly,

$$
H_{b}^{n}=\int_{0}^{\tau_{b}} \tilde{m}_{n}\left(B_{s}, \underline{B}_{s}\right) \mathrm{d} s .
$$

Using the uniform continuity of $\tilde{m}(u, v)$ on $\{(u, v): 1 / R \leq v \leq u \leq R\}$ for any $R \in(1, \infty)$, and the fact that $\sup _{0 \leq s \leq \tau_{b}} B_{s}(\omega)<\infty$ a.s., we know that, for any $\varepsilon>0$, there exists a $N=N(\omega)$ such that, for all $n>N(\omega)$, we have

$$
\begin{aligned}
\left|H_{b}-H_{b}^{n}\right| & =\left|\int_{0}^{\tau_{b}}\left[\tilde{m}\left(B_{s}, \underline{B}_{s}\right)-\tilde{m}_{n}\left(B_{s}, \underline{B}_{s}\right)\right] \mathrm{d} s\right| \\
& =\left|\int_{0}^{\tau_{b}}\left[\tilde{m}\left(B_{s}, \underline{B}_{s}\right)-\tilde{m}\left(B_{s}, \frac{1}{n}\left[n \underline{B}_{s}\right]\right)\right] \mathrm{d} s\right| \\
& \leq \varepsilon \tau_{b}
\end{aligned}
$$

and $\tau_{b}<\infty, \mathbb{P}_{x}$-a.s., so $H_{b} \rightarrow H_{b}^{n}$ a.s. Now, let

$$
\tilde{m}_{\min }(\omega)=\inf _{0 \leq s \leq \tau_{b}} \tilde{m}\left(B_{s}(\omega), \underline{B}_{s}(\omega)\right)<\infty \text { a.s. }
$$


By the definition of the inverse processes $A_{t}$ and $A_{t}^{n}$, we have

$$
\begin{aligned}
t \wedge H_{b} & =\int_{0}^{A_{t} \wedge \tau_{b}} \tilde{m}\left(B_{s}, \underline{B}_{s}\right) \mathrm{d} s \geq\left(A_{t} \wedge \tau_{b}\right) \tilde{m}_{\min }(\omega), \\
t \wedge H_{b}^{n} & =\int_{0}^{A_{t}^{n} \wedge \tau_{b}} \tilde{m}_{n}\left(B_{s}, \underline{B}_{s}\right) \mathrm{d} s .
\end{aligned}
$$

We first consider the case when $A_{t} \wedge \tau_{b} \leq A_{t}^{n} \wedge \tau_{b}$ (the other case is dealt with similarly). We know that $\sup _{0 \leq s \leq \tau_{b} \wedge A_{t}} B_{s}<\infty$ a.s. Subtracting (5.3) from (5.2), and again using the uniform continuity of $\tilde{m}$ in $m$, we see that

$$
\begin{aligned}
t \wedge H_{b}-t \wedge H_{b}^{n} & =\int_{0}^{A_{t} \wedge \tau_{b}}\left[\tilde{m}\left(B_{s}, \underline{B}_{s}\right)-\tilde{m}_{n}\left(B_{s}, \underline{B}_{s}\right)\right] \mathrm{d} s-\int_{A_{t} \wedge \tau_{b}}^{A_{t}^{n} \wedge \tau_{b}} \tilde{m}_{n}\left(B_{s}, \underline{B}_{s}\right) \mathrm{d} s \\
& \leq \varepsilon\left(A_{t} \wedge \tau_{b}\right)-\tilde{m}_{\min }(\omega)\left(A_{t}^{n} \wedge \tau_{b}-A_{t} \wedge \tau_{b}\right) \\
& \leq \frac{\varepsilon\left(t \wedge H_{b}^{n}\right)}{\tilde{m}_{\min }(\omega)}-\tilde{m}_{\min }(\omega)\left(A_{t}^{n} \wedge \tau_{b}-A_{t} \wedge \tau_{b}\right),
\end{aligned}
$$

where in the last line we have used the inequality in (5.2). Rearranging, we find that

$$
0 \leq \tilde{m}_{\min }\left(A_{t}^{n} \wedge \tau_{b}-A_{t} \wedge \tau_{b}\right) \leq \frac{\varepsilon\left(t \wedge H_{b}^{n}\right)}{\tilde{m}_{\min }}-\left(t \wedge H_{b}-t \wedge H_{b}^{n}\right) \quad \text { a.s. }
$$

But, we have already shown that $H_{b}^{n} \rightarrow H_{b}$ a.s, so the right-hand side can be made arbitrarily small, and, thus, $A_{t}^{n} \wedge \tau_{b} \rightarrow A_{t} \wedge \tau_{b}$ a.s. We proceed similarly for the case $A_{t}^{n} \wedge \tau_{b} \leq A_{t} \wedge \tau_{b}$. Then

$$
X_{t \wedge H_{b}}-X_{t \wedge H_{b}^{n}}^{n}=B_{A_{t} \wedge \tau_{b}}-B_{A_{t}^{n} \wedge \tau_{b}}
$$

and $B$ is continuous, so

$$
X_{t \wedge H_{b}}-X_{t \wedge H_{b}^{n}}^{n} \rightarrow 0 \quad \text { a.s. }
$$

as required.

\subsection{The occupation time formula}

Let $\left(l_{t}^{x}\right)$ denote the local time process for $B$ in (2.4) at the level $x$.

Theorem 5.1. Let $x=m, 0<\delta<x$, and $f: \mathbb{R}^{2} \mapsto \mathbb{R}^{+}$be a bounded, continuous function. Then we have the occupation time formula

$$
\int_{0}^{H_{\delta} \wedge t} f\left(X_{s}, \underline{X}_{s}\right) \mathrm{d} s=\sum_{\delta<m \leq x} \int_{m}^{\infty} f(x, m) \tilde{m}(x, m) l_{A_{t} \wedge \tau_{\delta}}^{x, m} \mathrm{~d} x \quad \text { a.s. },
$$

where $l_{t}^{x, m}=\int_{0}^{t} \mathbf{1}_{\left\{\underline{B}_{s} \in\{m\}\right\}} \mathrm{d} l_{s}^{x}=l_{\tau_{b-}}^{x}-l_{\tau_{b}}^{x} \geq 0$ is the local time that $B$ spends at $x$ when the minimum is exactly $m$, and the sum is taken over the (a.s. countable) $m$-values where $B$ makes a nonzero upward excursion from a minimum at $m$ (we know these $m$-values are a.s. countable from standard excursion theory for Brownian motion; see, e.g. [17, Chapter XII, Section 2]).

Proof. See Appendix A.

Remark 5.1. Theorem 5.1 is clearly more involved than the standard occupation time formula. However, it can be used to show that $\int_{0}^{\epsilon} \int_{0}^{u} \tilde{m}(u, v) \mathrm{d} v \mathrm{~d} u<\infty$ implies that

$$
\mathbb{P}_{x, m}\left(H_{0}<\infty\right)=1,
$$


which combined with Theorem 4.1 shows that $\mathbb{P}\left(H_{0}<\infty\right)$ is either 1 or 0 depending on the finiteness of $\int_{0}^{\epsilon} \int_{0}^{u} \tilde{m}(u, v) \mathrm{d} v \mathrm{~d} u$ (we defer the details to future work).

\section{Transition densities}

\subsection{Existence of a joint transition density for $\left(X_{t}, \underline{X}_{t}\right)$}

Theorem 6.1. Define the function

$$
\tilde{\sigma}(y, \underline{y})=\mathrm{e}^{-y} \sigma\left(\mathrm{e}^{y}, \mathrm{e}^{y}\right)
$$

for all $y \geq \underline{y}$, and assume that

- $\tilde{\sigma}(y, y)$ possesses bounded continuous partial derivatives of all orders up to and inclu $\bar{d}$ ing 2 ;

- $\int_{0}^{\varepsilon} \int_{0}^{u} \tilde{m}(u, v) \mathrm{d} v \mathrm{~d} u=\infty$ so $\mathbb{P}\left(H_{0}<\infty\right)=0$.

Then, under $\mathbb{P}_{x, x},\left(X_{t}, \underline{X}_{t}\right)$ defined in (2.1) admits a joint density $p_{t}\left(x^{\prime}, \underline{x}^{\prime}\right)$.

Remark 6.1. Note that, under $\mathbb{P}_{x, m}$ with $x>m$, there is a nonzero probability that $\underline{X}_{t}=$ $m \wedge \inf _{0 \leq s \leq t} X_{s}=m$, i.e. the law of $\underline{X}_{t}$ has an atom at $m$.

Proof of Theorem 6.1. Let $Y_{t}:=\log X_{t}$ and $\underline{Y}_{t}:=\log \underline{X}_{t}$, which are well defined because $X$ cannot hit 0 in finite time a.s. We note that $Y_{0}=\underline{Y}_{0}$. Using Itô's lemma, we have

$$
\mathrm{d} Y_{t}=\tilde{\sigma}\left(Y_{t}, \underline{Y}_{t}\right) \mathrm{d} W_{t}-\frac{1}{2} \tilde{\sigma}^{2}\left(Y_{t}, \underline{Y}_{t}\right) \mathrm{d} t .
$$

Let us define

$$
\rho_{t}=\inf \left\{u \leq t: X_{u}=\underline{X}_{t}\right\} .
$$

Because the log function is monotonically increasing, we have $\rho_{t}=\inf \left\{u \leq t: Y_{u}=\underline{Y}_{t}\right\}$. We now make a transformation of $Y$ to a process with diffusion coefficient equal to 1 . To this end, we first define

$$
\eta(\underline{y})=\int_{y_{0}}^{\underline{y}} \frac{\mathrm{d} u}{\tilde{\sigma}(u, u)}, \quad \beta(y, \underline{y})=\eta(\underline{y})+\int_{\underline{y}}^{y} \frac{\mathrm{d} u}{\tilde{\sigma}(u, \underline{y})},
$$

and consider the new processes $Z_{t}:=\beta\left(Y_{t}, \underline{Y}_{t}\right)$ and $\underline{Z}_{t}:=\inf _{s \leq t} Z_{s}$. Then $Z_{0}=\beta\left(Y_{0}, \underline{Y}_{0}\right)=0$. Note that, for all $t$,

$$
Z_{t}=\beta\left(Y_{t}, \underline{Y}_{t}\right)=\eta\left(\underline{Y}_{t}\right)+\int_{\underline{Y}_{t}}^{Y_{t}} \frac{\mathrm{d} u}{\tilde{\sigma}\left(u, \underline{Y}_{t}\right)} \geq \eta\left(\underline{Y}_{t}\right),
$$

and from this we see that

$$
\underline{Z}_{t}=\inf _{s \leq t} Z_{s} \geq \eta\left(\underline{Y}_{t}\right) .
$$

It turns out that we have equality in (6.1), since at time $\rho_{t} \leq t$ we have $Y_{\rho_{t}}=\underline{Y}_{t}$. Using the monotonicity of $\eta(\cdot)$ and $\beta(\cdot, \underline{y})$, we have

$$
\begin{aligned}
\underline{Y}_{t} & =\eta^{-1}\left(\underline{Z}_{t}\right), \\
Y_{t} & =\beta^{-1}\left(Z_{t}, \eta^{-1}\left(\underline{Z}_{t}\right)\right), \\
\rho_{t} & =\inf \left\{u \leq t: Z_{u}=\underline{Z}_{t}\right\},
\end{aligned}
$$

where $\beta^{-1}(\cdot, \underline{y})$ is the inverse of function $\beta(\cdot, \underline{y})$. 
Since $\beta$ is at least $C^{2}$, using Itô's lemma, we obtain

$$
\mathrm{d} Z_{t}=\mathrm{d} W_{t}-\frac{1}{2}\left[\tilde{\sigma}\left(Y_{t}, \underline{Y}_{t}\right)+\tilde{\sigma}_{y}\left(Y_{t}, \underline{Y}_{t}\right)\right] \mathrm{d} t=\mathrm{d} W_{t}+b\left(Z_{t}, \underline{Z}_{t}\right) \mathrm{d} t,
$$

where

$$
b(z, \underline{z})=-\frac{1}{2}\left[\tilde{\sigma}\left(\beta^{-1}\left(z, \eta^{-1}(\underline{z})\right), \eta^{-1}(\underline{z})\right)+\tilde{\sigma}_{y}\left(\beta^{-1}\left(z, \eta^{-1}(\underline{z})\right), \eta^{-1}(\underline{z})\right)\right] .
$$

In light of (6.2) and (6.3), it suffices to show that $\left(Z_{t}, \underline{Z}_{t}\right)$ has a density function.

We now mimic the proof of the main theorem in $[18$, p. 2$]$, and consider a new measure $\tilde{\mathbb{P}}$ defined by

$$
\left.\frac{\mathrm{d} \mathbb{P}}{\mathrm{d} \tilde{\mathbb{P}}}\right|_{\mathcal{F}_{t}}=\mathbb{E} p\left\{\int_{0}^{t} b\left(Z_{s}, \underline{Z}_{s}\right) \mathrm{d} Z_{s}-\frac{1}{2} \int_{0}^{t} b^{2}\left(Z_{s}, \underline{Z}_{s}\right) \mathrm{d} s\right\} .
$$

By Girsanov's theorem, the process $\left(Z_{t}\right)$ is a standard Brownian motion under the measure $\tilde{\mathbb{P}}$. Now define the $C^{2}$ function

$$
h(z, \underline{z})=\int_{\underline{z}}^{z} b(u, \underline{z}) \mathrm{d} u+\int_{0}^{\underline{z}} b(u, u) \mathrm{d} u .
$$

Using Itô's lemma, we have

$$
\mathrm{d} h\left(Z_{t}, \underline{Z}_{t}\right)=b\left(Z_{t}, \underline{Z}_{t}\right) \mathrm{d} Z_{t}+\frac{1}{2} b_{z}\left(Z_{t}, \underline{Z}_{t}\right) \mathrm{d} t,
$$

from which we obtain (note that $\left.h\left(Z_{0}, \underline{Z}_{0}\right)=h(0,0)=0\right)$ )

$$
h\left(Z_{t}, \underline{Z}_{t}\right)-\frac{1}{2} \int_{0}^{t} b_{z}\left(Z_{s}, \underline{Z}_{s}\right) \mathrm{d} s=\int_{0}^{t} b\left(Z_{s}, \underline{Z}_{s}\right) \mathrm{d} Z_{s} .
$$

Now, for any bounded bivariate continuous function $f$, we have

$$
\mathbb{E}\left(f\left(Z_{t}, \underline{Z}_{t}\right)\right)=\tilde{\mathbb{E}}\left(f\left(Z_{t}, \underline{Z}_{t}\right) \exp \left[h\left(Z_{t}, \underline{Z}_{t}\right)-\frac{1}{2} \int_{0}^{t} g\left(Z_{s}, \underline{Z}_{s}\right) \mathrm{d} s\right]\right),
$$

where $g=b^{2}+b_{z}$. Conditioning on $\left(Z_{t}, \underline{Z}_{t}\right)=(z, \underline{z})$ for $z>\underline{z}, \underline{z}<0$, we obtain

$$
\begin{aligned}
\mathbb{E}\left(f\left(Z_{t}, \underline{Z}_{t}\right)\right)=\int_{-\infty}^{0} \int_{\underline{z}}^{\infty} & f(z, \underline{z}) \phi_{t}(z, \underline{z}) \mathrm{e}^{h(z, \underline{z})} \\
& \times \tilde{\mathbb{E}}\left(\exp \left[-\frac{1}{2} \int_{0}^{t} g\left(Z_{s}, \underline{Z}_{s}\right) \mathrm{d} s\right] \mid Z_{t}=z, \underline{Z}_{t}=\underline{z}\right) \mathrm{d} z \mathrm{~d} \underline{z},
\end{aligned}
$$

where $\phi_{t}(z, \underline{z})$ is the joint density of the standard Brownian motion $\left(Z_{t}\right)$ and its minimum $\underline{Z}_{t}$. Thus, the pair $\left(Z_{t}, \underline{Z}_{t}\right)$ has joint density

$$
p_{t}^{Z, \underline{Z}}(z, \underline{z})=\phi_{t}(z, \underline{z}) \mathrm{e}^{h(z, \underline{z})} \tilde{\mathbb{E}}\left(\exp \left[-\frac{1}{2} \int_{0}^{t} g\left(Z_{s}, \underline{Z}_{s}\right) \mathrm{d} s\right] \mid Z_{t}=z, \underline{Z}_{t}=\underline{z}\right) .
$$

It follows that the pair $\left(Y_{t}, \underline{Y}_{t}\right)=\left(\log X_{t}, \log \underline{X}_{t}\right)$ has joint density

$$
p_{t}^{Y, \underline{Y}}(y, \underline{y})=p_{t}^{Z, \underline{Z}}(\beta(y, \underline{y}), \eta(\underline{y})) \frac{\partial \beta}{\partial y} \frac{\partial \eta}{\partial \underline{y}}=\frac{p_{t}^{Z, \underline{Z}}(\beta(y, \underline{y}), \eta(\underline{y}))}{\tilde{\sigma}(y, \underline{y}) \tilde{\sigma}(\underline{y}, \underline{y})} .
$$


Remark 6.2. For a stock price model of the form in (2.2), the existence of a semiclosed-form density for $\left(X_{t}, \underline{X}_{t}\right)$ as proved above allows us to price general barrier option contracts with payoffs of the form $\varphi\left(X_{t}, \underline{X}_{t}\right)$ for a measurable function $\varphi$.

\subsection{Characterizing the joint density in terms of Bessel-3 bridges}

From (6.5) and (6.6), it is seen that the regularity of the joint density of $p_{t}^{Y, \underline{Y}}(y, \underline{y})$ depends on that of $h$ in (6.4) and the following function $\psi_{t}$ :

$$
\psi_{t}(z, \underline{z})=\tilde{\mathbb{E}}\left(\exp \left[-\frac{1}{2} \int_{0}^{t} g\left(Z_{s}, \underline{Z}_{s}\right) \mathrm{d} s\right] \mid Z_{t}=z, \underline{Z}_{t}=\underline{z}\right) .
$$

The function $\psi_{t}$ depends on the law of a standard Brownian motion $\left(Z_{s}\right)_{0 \leq s \leq t}$ given $Z_{t}$, and $\underline{Z}_{t}$. To this end, let us condition on $\left(Z_{t}, \underline{Z}_{t}, \rho_{t}\right)=(z, \underline{z}, u) ;\left(Z_{t}, \underline{Z}_{t}, \rho_{t}\right)$ has a smooth density given by

$$
\chi_{t}(z, \underline{z}, u)=2 f(\underline{z}, u) f(\underline{z}-z, t-u)=\frac{-\underline{z}(z-\underline{z})}{\pi u^{3 / 2}(t-u)^{3 / 2}} \exp \left[-\frac{\underline{z}^{2}}{2 u}-\frac{(z-\underline{z})^{2}}{2(t-u)}\right],
$$

where $f(y, t)=|y| \mathrm{e}^{-y^{2} / 2 t} / \sqrt{2 \pi t^{3}}$ is the hitting time density from 0 to $y$ for a standard Brownian motion (see, e.g. [11]). Moreover, given $\left(Z_{t}, \underline{Z}_{t}, \rho_{t}\right)=(z, \underline{z}, u)$, the path fragments

$$
\left(Z_{u-s}-\underline{z}\right)_{0 \leq s \leq u} \quad \text { and } \quad\left(Z_{u+s}-\underline{z}\right)_{0 \leq s \leq t-u}
$$

are two independent Brownian meanders of lengths $u$ and $t-u$, starting at 0 and conditioned to end at $-\underline{z}>0$ and $z-\underline{z}>0$, respectively (see, e.g. [2]). A Brownian meander of length $s$ is defined as the rescaled portion of a Brownian path following the last passage time at 0 , $G_{1}=\sup \left\{s \leq 1: B_{s}=0\right\}$ :

$$
B_{u}^{\mathrm{me}}=\frac{\sqrt{s}}{\sqrt{1-G_{1}}}\left|B_{G_{1}+u\left(1-G_{1}\right) / s}\right| \quad 0 \leq u \leq s
$$

(see [3, p. 63]). It is known that the law of a Brownian meander of length $s$ is identical to that of a standard Brownian motion starting at 0 and conditioned to be positive for $t \in[0, s]$ (see, e.g. [8]). Moreover, the tied-down Brownian meander, i.e. the Brownian meander conditioned so that $B_{1}^{\mathrm{me}}=x>0$, has the same law as a three-dimensional Bessel bridge $R^{\mathrm{br}}$ with $R_{0}^{\text {br }}=0$ and $R_{1}^{\text {br }}=x$ (see, e.g. [1] and [11]). Hence, the path fragments $\left(Z_{u-s}-\underline{z}\right)_{0 \leq s \leq u}$ and $\left(Z_{u+s}-\underline{z}\right)_{0 \leq s \leq t-u}$ can be identified with two independent Bessel-3 bridges, starting at 0 , and ending at $-\underline{z}>0$ and $z-\underline{z}>0$, respectively (see [2] and [23]). Thus, as in [16], we have

$$
\begin{aligned}
\kappa_{t}(z, \underline{z}, u)= & \tilde{\mathbb{E}}\left(\exp \left[-\frac{1}{2} \int_{0}^{u} g\left(Z_{s}, \underline{Z}_{s}\right) \mathrm{d} s\right] \mid Z_{t}=z, \underline{Z}_{t}=\underline{z}, \rho_{t}=u\right) \\
& \times \tilde{\mathbb{E}}\left(\exp \left[-\frac{1}{2} \int_{u}^{t} g\left(Z_{s}, \underline{z}\right) \mathrm{d} s\right] \mid Z_{t}=z, \underline{Z}_{t}=\underline{z}, \rho_{t}=u\right) \\
= & \tilde{\mathbb{E}}\left(\exp \left[-\frac{1}{2} \int_{0}^{u} g\left(Z_{s}, \underline{Z}_{s}\right) \mathrm{d} s\right] \mid Z_{t}=z, \underline{Z}_{t}=\underline{z}, \rho_{t}=u\right) \\
& \times \tilde{\mathbb{E}}\left(\exp \left[-\frac{1}{2} \int_{0}^{t-u} g\left(Z_{t-s}, \underline{z}\right) \mathrm{d} s\right] \mid Z_{t}=z, \underline{Z}_{t}=\underline{z}, \rho_{t}=u\right),
\end{aligned}
$$


and we can rewrite the last expectation in terms of the two aforementioned independent Bessel-3 bridges if we wish. It follows that

$$
\begin{aligned}
\psi_{t}(z, \underline{z}) & =\tilde{\mathbb{E}}\left(\exp \left[-\frac{1}{2} \int_{0}^{t} g\left(Z_{s}, \underline{Z}_{s}\right) \mathrm{d} s\right] \mid Z_{t}=z, \underline{Z}_{t}=\underline{z}\right) \\
& =\int_{0}^{t} \kappa_{t}(z, \underline{z}, u) \tilde{\mathbb{P}}\left(\rho_{t} \in \mathrm{d} u \mid Z_{t}=z, \underline{Z}_{t}=\underline{z}\right) \mathrm{d} u \\
& =\int_{0}^{t} \kappa_{t}(z, \underline{z}, u) \frac{\chi_{t}(z, \underline{z}, u)}{\phi_{t}(z, \underline{z})} \mathrm{d} u .
\end{aligned}
$$

\section{A generalized forward Kolmogorov equation}

In this section we assume that $m=x=x_{0}$, so $X_{0}=\underline{X}_{0}=x_{0}>0$ and we use $\mathbb{E}$ as shorthand for $\mathbb{E}_{x_{0}, x_{0}}$. We further assume that $\int_{0}^{\varepsilon} \int_{0}^{u} \tilde{m}(u, v) \mathrm{d} v \mathrm{~d} u=\infty$, so $\mathbb{P}_{x, x}\left(H_{0}<\infty\right)=0$, i.e. $X$ cannot hit 0 a.s., and, for simplicity, we assume that $\sigma$ is bounded (we can easily relax this assumption by working in $\log$ space as in the previous section, but in the interests of clarity and succinctness, we do not do this here). Let $\mathcal{O}=\left\{(x, y) \in \mathbb{R}^{+} \times \mathbb{R}^{+}: x \geq y\right\}$ denote the support of $\left(X_{t}, \underline{X}_{t}\right)$.

Theorem 7.1. $\left(X_{t}, \underline{X}_{t}\right)$ satisfies the following forward equation

$$
\frac{\partial}{\partial t} \mathbb{E}\left(f\left(X_{t}, \underline{X}_{t}, t\right)\right)=\mathbb{E}\left(f_{t}\left(X_{t}, \underline{X}_{t}, t\right)+\frac{1}{2} f_{x x}\left(X_{t}, \underline{X}_{t}, t\right) \sigma\left(X_{t}, \underline{X}_{t}\right)^{2}\right)
$$

for all test functions $f \in C_{b}^{2,1,1}\left(\mathcal{O} \times \mathbb{R}^{+}\right)$satisfying $f_{y}(y, y, t)=0$.

Proof. See Appendix B.

Remark 7.1. If $f \in C_{c}^{\infty}\left(\mathcal{O} \times \mathbb{R}^{+}\right)\left(C_{c}^{\infty}\right.$ means smooth with compact support), re-writing (7.1) in terms of integrals and integrating from $t=0$ to $\infty$, and using the fact that $f\left(t, X_{t}, \underline{X}_{t}\right)=0$ a.s. for sufficiently large $t$, we see that $p(t, \mathrm{~d} x, \mathrm{~d} y)=\mathbb{P}\left(X_{t} \in \mathrm{d} x, \underline{X}_{t} \in \mathrm{d} y\right)$ satisfies

$$
\int_{t=0}^{\infty} \int_{\mathcal{O}}\left(f_{t}+\frac{1}{2} \sigma(x, y)^{2} f_{x x}\right) p(t, \mathrm{~d} x, \mathrm{~d} y) \mathrm{d} t=0 .
$$

Remark 7.2. If $p(t, \mathrm{~d} x, \mathrm{~d} y)$ admits a density so that $p(t, \mathrm{~d} x, \mathrm{~d} y)=p(t, x, y) \mathrm{d} x \mathrm{~d} y$, and $p$ and $\sigma$ are twice continuously differentiable in $x$ and $p$ is once differentiable in $t$, then integrating (7.2) by parts we have

$$
\int_{t=0}^{\infty} \int_{\mathcal{O}} f(x, y, t)\left[-\partial_{t} p+\partial_{x x}^{2}\left(\frac{1}{2} \sigma(x, y)^{2} p\right)\right] \mathrm{d} x \mathrm{~d} y \mathrm{~d} t=0,
$$

and, thus (by the arbitraryness of $f$ ), $p(t, x, y)$ is a classical solution to the family of forward Kolmogorov equations

$$
\partial_{t} p=\partial_{x x}^{2}\left(\frac{1}{2} \sigma(x, y)^{2} p\right), \quad x \neq y,
$$

for all $y \leq x$ (see [21, p. 252], Theorem 3.2.6 of [22], and [9] for similar results and weak formulations for a standard diffusion process). 


\subsection{A forward equation for down-and-out call options}

Proposition 7.1. Assume that $k>0$ and $0<b<x_{0}$. Then

$$
\mathbb{E}\left(\left(X_{t}-k\right)^{+} \mathbf{1}_{\left\{\underline{X}_{t}>b\right\}}\right)-\left(X_{0}-k\right)^{+}=\frac{1}{2} \mathbb{E}\left(L_{t \wedge H_{b}}^{k}\right)-(b-k)^{+} \mathbb{P}\left(\underline{X}_{t} \leq b\right),
$$

where $L_{t}^{a}$ is the semimartingale local time of $X$ at a as defined in, e.g. Theorem 3.7.1 of [12] and $H_{b}=\inf \left\{s: X_{s}=y\right\}$, subject to the following boundary condition at $x=y$ :

$$
\mathbb{E}\left(\left(X_{t}-b\right)^{+} \mathbf{1}_{\left\{\underline{X}_{t}>b\right\}}\right)=\mathbb{E}\left(\left(X_{t}-b\right) \mathbf{1}_{\left\{\underline{X}_{t}>b\right\}}\right)=x_{0}-b .
$$

Remark 7.3. Equation (7.3) is a forward equation for a down-and-out call option on $X_{t}$ with strike $x$, which knocks out if $X$ hits $y$ before time $t$. Specifically (assuming zero interest rates and dividends), the left-hand side is the fair price of the down-and-out call, and the $\mathbb{P}\left(\underline{X}_{t} \leq y\right)$ term on the right-hand side is the price of a one-touch option on $\underline{X}_{t}$ which pays 1 if $X$ hits $y$ before $t$.

Remark 7.4. Equation (7.4) is the same condition that appears in [20], and if $\underline{X}_{t}$ has no atom at $y$, we can differentiate (7.4) with respect to $y$ to obtain the condition in Theorem 3.1 of [19].

Remark 7.5. The financial interpretation of (7.4) is the well-known result that (for zero dividends and interest rates) we can semistatically hedge a down-and-out call option with barrier $b$ equal to the strike $k$, by buying one unit of stock and holding $-b$ dollars, and unwinding the position if/when the barrier is struck (see, e.g. Appendix A of [7]).

Proof of Proposition 7.1. From the generalized Itô formula given in, e.g. Theorem 3.7.1 of [12], we obtain

$$
\mathrm{d}\left(X_{t}-k\right)^{+}=\mathbf{1}_{\left\{X_{t}>k\right\}} \mathrm{d} X_{t}+\frac{1}{2} \mathrm{~d} L_{t}^{k} .
$$

Integrating from time 0 to $t \wedge H_{b}$ we obtain

$$
\begin{aligned}
\left(X_{t \wedge H_{b}}-k\right)^{+}-\left(X_{0}-k\right)^{+} & =\left(X_{t}-k\right)^{+} \mathbf{1}_{\left\{H_{b}>t\right\}}+(b-k)^{+} \mathbf{1}_{\left\{H_{b} \leq t\right\}}-\left(X_{0}-k\right)^{+} \\
& =\int_{0}^{t \wedge H_{b}} \mathbf{1}_{\left\{X_{s}>x\right\}} \mathrm{d} X_{s}+\frac{1}{2} L_{t \wedge H_{b}}^{k} .
\end{aligned}
$$

Taking expectations and simplifying, we obtain (7.3).

To obtain the boundary condition in (7.4), we use the optional sampling theorem for the bounded stopping time $t \wedge H_{b}$ to obtain

$$
\begin{aligned}
\mathbb{E}\left(X_{t \wedge H_{b}}\right) & =x_{0} \\
& =\mathbb{E}\left(X_{t} \mathbf{1}_{\left\{\underline{X}_{t}>b\right\}}\right)+\mathbb{E}\left(X_{H_{b}} \mathbf{1}_{\left\{H_{b} \leq t\right\}}\right) \\
& =\mathbb{E}\left(X_{t} \mathbf{1}_{\left\{\underline{X}_{t}>b\right\}}\right)+b \mathbb{P}\left(\underline{X}_{t} \leq b\right) \\
& =\mathbb{E}\left(\left(X_{t}-b\right) \mathbf{1}_{\left\{\underline{X}_{t}>b\right\}}\right)+b \mathbb{E}\left(\mathbf{1}_{\left\{\underline{X}_{t}>b\right\}}\right)+b \mathbb{E}\left(\mathbf{1}_{\left\{\underline{X}_{t} \leq b\right\}}\right) \\
& =\mathbb{E}\left(\left(X_{t}-b\right) \mathbf{1}_{\left\{\underline{X}_{t}>b\right\}}\right)+b \\
& =\mathbb{E}\left(\left(X_{t}-b\right)^{+} \mathbf{1}_{\left\{\underline{X}_{t}>b\right\}}\right)+b,
\end{aligned}
$$

where the last equality follows because $X_{t}>b$ on $\left\{\underline{X}_{t}>b\right\}$, i.e. if $X$ does not hit $b$ before time $t$.

\section{Appendix A. Proof of Theorem 5.1}

The process $\left(X_{t}^{n}\right)$ defined in (5.1) is just a regular one-dimensional diffusion process for $t \in\left[H_{(k+1) / n}^{n}, H_{k / n}^{n}\right)$ for each $k=0, \ldots,\left[x_{0} n\right]-1$. Using the standard occupation time 
formula for $t \in\left[H_{(k+1) / n}^{n}, H_{k / n}^{n}\right.$ ) for each $k$ (see Theorem 49.1 of [21]), we have

$$
\begin{aligned}
\int_{H_{(k+1) / n}^{n} \wedge t}^{H_{k / n}^{n} \wedge t} f_{n}\left(X_{s}^{n}, \underline{X}_{S}^{n}\right) \mathrm{d} s & =\int_{\delta}^{\infty} f\left(x, \frac{k}{n}\right) \tilde{m}\left(x, \frac{k}{n}\right) l_{A_{t}^{n} \wedge \tau_{\delta}}^{x,(k / n,(k+1) / n]} \mathrm{d} x \\
& =\int_{\delta}^{\infty} \sum_{k / n<m \leq(k+1) / n} f_{n}(x, m) \tilde{m}_{n}(x, m) l_{A_{t}^{n} \wedge \tau_{\delta}}^{x, m} \mathrm{~d} x,
\end{aligned}
$$

where $f_{n}(x, m)=f(x,[n m] / n), l_{t}^{x,(a, b]}=\int_{0}^{t} \mathbf{1}_{\left\{\underline{B}_{s} \in(a, b]\right\}} \mathrm{d} l_{s}^{x}$ is the local time that $B$ has accrued at $x$ at time $t$ while $\underline{B} \in(a, b]$, and we are summing over (a.s. countable) $m$-values in $(k / n,(k+1) / n]$ for which there is a nonzero upward excursion from a minimum at $m$.

Summing over $k$ until time $t \wedge H_{\delta}^{n}$ and taking the finite sum inside the integral on the right-hand side, we obtain

$$
\begin{aligned}
\int_{0}^{t \wedge H_{\delta}^{n}} f\left(X_{s}^{n}, \underline{X}_{s}^{n}\right) \mathrm{d} s & =\int_{0}^{t} f\left(X_{s}^{n}, \underline{X}_{s}^{n}\right) \mathbf{1}_{\left\{s<H_{\delta}^{n}\right\}} \mathrm{d} s \\
& =\sum_{k=0}^{\left[x_{0} n\right]-1} \int_{\delta}^{\infty} \sum_{k / n<m \leq(k+1) / n} f_{n}(x, m) \tilde{m}_{n}(x, m) l_{A_{t}^{n} \wedge \tau_{\delta}}^{x, m} \mathrm{~d} x \\
& =\int_{\delta}^{\infty}\left[\sum_{\delta<m \leq x} f_{n}(x, m) \tilde{m}_{n}(x, m) l_{A_{t}^{n} \wedge \tau_{\delta}}^{x, m}\right] \mathrm{d} x \\
& =\int_{\delta}^{\sup _{0 \leq s \leq \tau_{\delta}} B_{s}}\left[\sum_{\delta<m \leq x} f_{n}(x, m) \tilde{m}_{n}(x, m) l_{A_{t}^{n} \wedge \tau_{\delta}}^{x, m}\right] \mathrm{d} x .
\end{aligned}
$$

For the left-hand integral, from Proposition 5.1, we know that $H_{\delta}^{n} \rightarrow H_{\delta}$ a.s. and $X_{t \wedge H_{\delta}^{n}}^{n} \rightarrow$ $X_{t \wedge H_{\delta}}$ a.s., so $f\left(X_{s}^{n}, \underline{X}_{s}^{n}\right) \mathbf{1}_{\left\{s<H_{\delta}^{n}\right\}} \rightarrow f\left(X_{s}, \underline{X}_{s}\right) \mathbf{1}_{\left\{s<H_{\delta}\right\}}$ Lebesgue almost everywhere on [0,t], a.s. Thus, by the dominated convergence theorem, we have

$$
\int_{0}^{t} \mathbf{1}_{\left\{s \leq H_{\delta}^{n}\right\} f\left(X_{s}^{n}, \underline{X}_{s}^{n}\right) \mathrm{d} s} \rightarrow \int_{0}^{t} \mathbf{1}_{\left\{s \leq H_{\delta}\right\}} f\left(X_{s}, \underline{X}_{s}\right) \mathrm{d} s=\int_{0}^{t \wedge H_{\delta}} f\left(X_{s}, \underline{X}_{s}\right) \mathrm{d} s \quad \text { a.s. }
$$

For the integrand on the right-hand side, we have the upper bound

$$
\sum_{\delta<m \leq x} f_{n}(x, m) \tilde{m}_{n}(x, m) l_{A_{t}^{n} \wedge \tau_{\delta}}^{x, m} \leq f_{\max } \tilde{m}_{\max }(\delta, \omega) l_{A_{t}^{n} \wedge \tau_{\delta}}^{x}<\infty \quad \text { a.s. }
$$

where $\tilde{m}_{\max }(\delta, \omega)=\sup _{0 \leq s \leq \tau_{\delta}} \tilde{m}\left(B_{s}, \underline{B}_{s}\right)<\infty$ a.s. Thus, letting $n \rightarrow \infty$ on both sides of (A.1), and applying the dominated convergence theorem to the right-hand side as well, and then applying Fubini's theorem, we obtain (5.4).

\section{Appendix B. Proof of Theorem 7.1}

Let $\sigma_{t}=\sigma\left(X_{t}, \underline{X}_{t}\right) ; X_{t}$ and $\underline{X}_{t}$ are continuous semimartingales, so we can apply Itô's formula to the test function $f \in C_{b}^{2,1,1}\left(\mathcal{O} \times \mathbb{R}^{+}\right)$:

$$
\begin{aligned}
\mathrm{d} f\left(X_{t}, \underline{X}_{t}, t\right) & =f_{x}\left(X_{t}, \underline{X}_{t}, t\right) \mathrm{d} X_{t}+\frac{1}{2} f_{x x}\left(X_{t}, \underline{X}_{t}, t\right) \sigma_{t}^{2} \mathrm{~d} t+f_{y}\left(\underline{X}_{t}, \underline{X}_{t}, t\right) \mathrm{d} \underline{X}_{t}, \\
& =f_{x}\left(X_{t}, \underline{X}_{t}, t\right) \mathrm{d} X_{t}+\frac{1}{2} f_{x x}\left(X_{t}, \underline{X}_{t}, t\right) \sigma_{t}^{2} \mathrm{~d} t .
\end{aligned}
$$


Here we have used the fact that $X_{t}=\underline{X}_{t}$ on the growth set of $\underline{X}_{t}$ in the final term (by growth set we mean the support of the random measure induced by the process $Y$ on $[0, T]$, i.e. the complement of the largest open set of zero measure). (Recall that $\psi_{y}(y, y, t)=0$.) Integrating we obtain

$$
f\left(X_{t}, \underline{X}_{t}, t\right)-f\left(x_{0}, x_{0}, 0\right)=\int_{0}^{t} f_{x}\left(X_{s}, \underline{X}_{s}, s\right) \mathrm{d} X_{s}+\int_{0}^{t} \frac{1}{2} f_{x x}\left(X_{s}, \underline{X}_{s}, s\right) \sigma_{s}^{2} \mathrm{~d} s .
$$

Taking expectations, and applying Fubini's theorem yields

$$
\mathbb{E}\left(f\left(X_{t}, \underline{X}_{t}, t\right)\right)-f\left(x_{0}, x_{0}, 0\right)=\int_{0}^{t} \frac{1}{2} \mathbb{E}\left(f_{x x}\left(X_{s}, \underline{X}_{s}, s\right) \sigma_{s}^{2}\right) \mathrm{d} s .
$$

Since $X_{t}$ and $\underline{X}_{t}$ are continuous in $t$ a.s. and $\sigma(\cdot, \cdot)$ is continuous, $\sigma_{t}=\sigma\left(X_{t}, \underline{X}_{t}, t\right)$ is also continuous in $t$ a.s. Moreover, $f \in C_{b}^{2,1,1}$, so $f_{x x}(\cdot, \cdot)$ is bounded and continuous, and $f_{x x}\left(X_{u}, \underline{X}_{u}, u\right) \sigma_{u}^{2} \rightarrow f_{x x}\left(X_{s}, \underline{X}_{s}, s\right) \sigma_{s}^{2}$ a.s. as $u \rightarrow s$. As $\sigma$ is also bounded, from the dominated convergence theorem we have

$$
\lim _{u \rightarrow s} \mathbb{E}\left(f_{x x}\left(X_{u}, \underline{X}_{u}, u\right) \sigma_{u}^{2}\right)=\mathbb{E}\left(f_{x x}\left(X_{s}, \underline{X}_{s}, s\right) \sigma_{s}^{2}\right),
$$

so the integrand $\mathbb{E}\left(f_{x x}\left(X_{s}, \underline{X}_{s}, s\right) \sigma_{s}^{2}\right)$ in (B.1) is continuous in $s$ for all $s$. Thus, using the fundamental theorem of calculus, we can differentiate (B.1) everywhere with respect to $t$ to get

$$
\frac{\partial}{\partial t} \mathbb{E}\left(f\left(X_{t}, \underline{X}_{t}, t\right)\right)=\mathbb{E}\left(f_{t}\left(X_{t}, \underline{X}_{t}, t\right)+\frac{1}{2} f_{x x}\left(X_{t}, \underline{X}_{t}, t\right) \sigma\left(X_{t}, \underline{X}_{t}\right)^{2}\right) .
$$

\section{Acknowledgements}

The authors would like to thank Gerard Brunick and Chris Rogers for sharing many useful insights.

\section{References}

[1] Bertoin, J., Chaumont, L. And Pitman, J. (2003). Path transformations of first passage bridges. Electron. Commun. Prob. 8, 155-166.

[2] Bertoin, J., Pitman, J., And Ruiz de Chavez, J. (1999). Constructions of a Brownian path with a given minimum. Electron. Commun. Prob. 4, 31-37.

[3] Borodin, A. N. and SAlminen, P. (2002). Handbook of Brownian Motion-Facts and Formulae, 2nd edn. Birkhäuser, Basel.

[4] Brunick, G. AND Shreve, S. (2012). Matching an Itô process by a solution of a stochastic differential equation. Submitted.

[5] CARr, P. (2009). Local variance gamma option pricing model. Presentation, Bloomberg/Courant Institute.

[6] Cox, A. M. G., Hobson, D. And ObŁós, J. (2011). Time-homogeneous diffusions with a given marginal at a random time. ESAIM Prob. Statist. 15, S11-S24.

[7] Dermann, E., Ergener, D. and Kani, I. (1995). Static options replication. J. Derivatives 2, 78-95.

[8] Durrett, R. T., Iglehart, D. L. And Miller, D. R. (1977). Weak convergence to Brownian meander and Brownian excursion. Ann. Prob. 5, 117-129.

[9] Figalli, A. (2008). Existence and uniqueness of martingale solutions for SDEs with rough or degenerate coefficients. J. Funct. Anal. 254, 109-153.

[10] Forde, M. (2011). A diffusion-type process with a given joint law for the terminal level and supremum at an independent exponential time. Stoch. Process. Appl. 121, 2802-2817.

[11] IмноF, J.-P. (1984). Density factorizations for Brownian motion, meander and the three dimensional Bessel process, and applications. J. Appl. Prob. 21, 500-510.

[12] Karatzas, I. And Shreve, S. E. (1991). Brownian Motion and Stochastic Calculus, 2nd edn. Springer, New York. 
[13] Karlin, S. and Taylor, H. M. (1981). A Second Course in Stochastic Processes. Academic Press, New York.

[14] Mao, X. (1997). Stochastic Differential Equations and Their Applications. Horwood Publishing, Chichester.

[15] Mohammed, S. E. A. (1984). Stochastic Functional Differential Equations. Pitman, Boston, MA.

[16] Pauwels, E. J. (1987). Smooth first-passage densities for one-dimensional diffusions. J. Appl. Prob. 24, 370-377.

[17] Revuz, D. And Yor, M. (1999). Continuous Martingales and Brownian Motion, 3rd edn. Springer, Berlin.

[18] Rogers, L. C. G. (1985). Smooth transition densities for one-dimensional diffusions. Bull. London Math. Soc. 17, 157-161.

[19] Rogers, L. C. G. (1993). The joint law of the maximum and the terminal value of a martingale. Prob. Theory Relat. Fields 95, 451-466.

[20] Rogers, L. C. G. (2012). Extremal martingales. Talk at EPSRC Symposium Workshop - Optimal Stopping, Optimal Control and Finance.

[21] Rogers, L. C. G. And Williams, D. (1987). Diffusions, Markov Processes and Martingales, Vol. 2. John Wiley, New York.

[22] Stroock, D. W. and Varadhan, S. R. S. (1979). Multidimensional Diffusion Processes. Springer, Berlin.

[23] Williams, D. (1974). Path decomposition and continuity of local time for one-dimensional diffusions. I. Proc. London Math. Soc. 28, 738-768.

[24] Williams, D. (1991). Probability with Martingales. Cambridge University Press. 